\section{Entscheidungshilfe für das frühe Cochleaimplantat}

Für die möglichst altersentsprechende Sprachentwicklung sind die ersten Lebensjahre von großer Bedeutung. Wie gut die Ergebnisse einer frühen Versorgung gehörloser oder schwer gehörgeschädigter Kinder mit einem Cochleaimplantat sind, wurde in einer italienischen Studie untersucht.

n einer Untersuchung mit 185 Kindern mit einem Cochleaimplantat (CI) sowie 34 mit einem Hirnstammimplantat sollte gezeigt werden, wie sinnvoll eine frühzeitige Implantatversorgung ist. Die Kinder wurden je nach Zeitpunkt der Implantation (von 4-36 Monaten) in drei

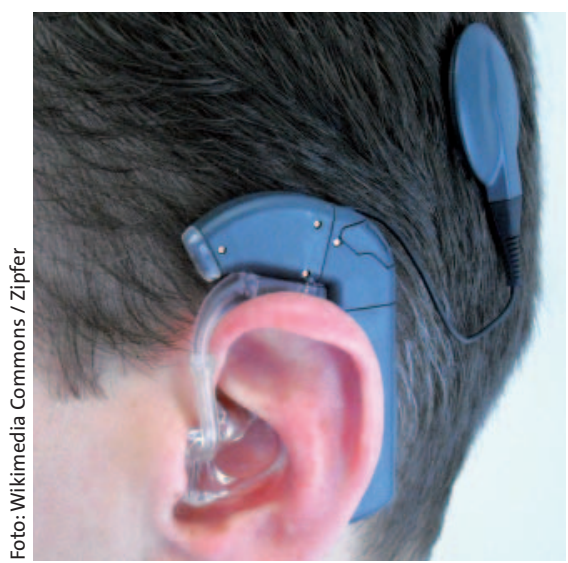

Cochleaimplantat: je früher, desto besser
Gruppen eingeteilt. 13 Kinder hatten ihr Implantat (immer dasselbe Modell) bereits in einem Alter von unter zwölf Monaten erhalten, in keinem Fall kam es zu Komplikationen. Zur Bestimmung der Sprachentwicklung wurden die auditorische Kategorie (CAP), der Peabody-Bild-Vokabel-Test in revidierter Form (PPVT-R), der TROG-Test zum grammatikalischen Verständnis und das SprachverständnisRating (SIR) angewandt.

In allen drei Gruppen wurden teils sehr gute CAP-Ergebnisse erreicht, allerdings verbesserte sich die auditorische Kategorie nach CI-Versorgung bei den jüngsten Patienten besonders rasch nach der Operation und erreichte bereits nach $24 \mathrm{Mo}$ naten einen Wert von 7 , signifikant früher als in den anderen Gruppen.

Der PPVT-R zeigte eine weitgehende Normalisierung des rezeptiven Sprachvermögens nur für die Kinder, die im ersten Lebensjahr ein CI erhalten hatten. Kinder, die erst mit 12-23 oder 24-36 Monaten mit einem Implantat versorgt worden waren, erreichten auch bis zu neun Jahre nach dem Eingriff nicht die Ergebnisse gleichaltriger gesunder Kinder. Das grammatikalische Verständnis hatte sich von den am frühesten versorgten Kindern nach fünf Jahren bei $77 \%$ bis in die 76-100. Perzentile hinein entwickelt. Nach neun Jahren lagen alle Kinder mit früher Implantatversorgung in diesem Bereich, aber nur $38 \%$ derjenigen, die das Implantat im zweiten und $20 \%$ derjenigen, die es im dritten Lebensjahr erhalten hatten.

Kategorie 5 im Sprachverständlichkeitsindex SIR erreichten nach fünf Jahren alle Kinder der ersten, $80 \%$ der zweiten und $63 \%$ der dritten Gruppe. Nach neun Jahren hatten sich die Ergebnisse überall weiter normalisiert, in Kategorie 3 fielen noch 10\% der Kinder aus Gruppe 3.

Fazit: Eine CI-Versorgung im ersten Lebensjahr ermöglicht eine Normalisierung der audio-phonologischen Parameter mit einer rasch praktisch altersgerechten Verständnis- und Sprachentwicklung, ohne dass es vermehrt zu Komplikationen kommen muss.

fk

Colletti L. Long-term follow-up of infants (4-11 months) fitted with cochlear implants. Acta Oto-Laryngologica 2009;129:361-6

\section{Grenzen der Tympanometrie beachten}

\section{Bei Trommelfellretraktion oder Trommelfellfalten verändert sich das} Ergebnis der Tympanometrie. In diesen Fällen ist die Methode wahrscheinlich nicht geeignet zur Bestimmung des Mittelohrdrucks.

D ie Tympanometrie misst primär nicht den Mittelohrduck, sondern die Energieübertragung auf das Mittelohr. Damit ist die Messung nicht nur abhängig vom Mittelohrdruck, sondern auch von der Form und Beschaffenheit des Trommelfells. Wie sich der Spitzenwert im Tympanogramm in Abhängigkeit von der Retraktion des Tympanums oder Falten und Taschen im Trommelfell verändert, untersuchten Rostocker HNO-Ärzte an einem einfachen Modell. Zwischen zwei 5- $\mathrm{cm}^{3}$-Spritzen, die den Ohrkanal und das Mittelohr repräsentierten, spannten sie luftdicht als Trommelfell verschiedene per Hand präparierte Gummimembranen aus Operationshandschuhen, die teils nur retrahiert, teils mit Taschen und Ausbuchtungen versehen wurden.

Je nachdem, wie das künstliche Trommelfell verändert war, verschob sich im Tympanogramm der Peak: Wies eine Retraktionstasche in den Mittelohrraum hinein, verschob sich der Spitzenwert in den negativen, bei einer Orientierung nach außen in den positiven Bereich. Dabei waren die Veränderungen in der Tympanometrie auch von der Lage der Ausbuchtungen und deren Größe abhängig. Der Spitzenwert verschob sich umso deut- licher, je geringer das Volumen hinter dem Trommelfell angelegt wurde. Die Autoren betonten, dass dies nicht nur eine Folge einer Hysterese ist, sondern die Luftmenge hinter dem Trommelfell repräsentiert, die den Spielraum der Trommelfellschwingung reduziert.

Fazit: Aus ihrem einfachen Modell schließen die Autoren, dass die Tympanometrie bei retrahiertem Trommelfell und Ausbuchtungen oder Falten des Tympanums nicht geeignet ist, um den Mittelohrdruck zu ermitteln. Das Ergebnis ist je nach Veränderung zu unterschiedlich. $\quad \mathbf{f k}$

Pau HW et al. Tympanometric experiments on retracted ear drums - does tympanometry reflect the true middle ear pressure? Acta Oto-Laryngologica 2009; 129: 1080-7 